\title{
The efficacy of gabapentin combined with opioids for neuropathic cancer pain: a meta-analysis
}

\author{
Hainan Bao ${ }^{1}$, Zhouyue $\mathrm{Wu}^{1}$, Qinxin Wang ${ }^{1}$, Jiatong Wang ${ }^{1}$, Li Zhang ${ }^{2}$, Lingtong Meng ${ }^{1}$, Feng Han ${ }^{1}$ \\ ${ }^{1}$ Key Laboratory of Cardiovascular \& Cerebrovascular Medicine, School of Pharmacy, Nanjing Medical University, Nanjing, China; ${ }^{2}$ Department of \\ Geriatric Neurology, Nanjing Brain Hospital Affiliated to Nanjing Medical University, Nanjing, China \\ Contributions: (I) Conception and design: F Han, L Meng, H Bao, Z Wu, Q Wang; (II) Administrative support: F Han, L Meng; (III) Provision of \\ study materials or patients: L Zhang; (IV) Collection and assembly of data: H Bao, J Wang, Z Wu; (V) Data analysis and interpretation: H Bao, \\ J Wang, Z Wu; (VI) Manuscript writing: All authors; (VII) Final approval of manuscript: All authors. \\ Correspondence to: Feng Han, PhD; Lingtong Meng, PhD. Key Laboratory of Cardiovascular \& Cerebrovascular Medicine, School of Pharmacy, \\ Nanjing Medical University, Nanjing 211166, China. Email: fenghan169@njmu.edu.cn; mlt@njmu.edu.cn.
}

\begin{abstract}
Background: More than 30\% of cancer patients experience neuropathic pain. Opioids, as standard painrelief agents, cannot achieve satisfactory outcomes to treat neuropathic cancer pain due to drug resistance and side effects. Meanwhile, gabapentin, a third-generation anticonvulsant drug, has great potential in providing relief for neuropathic cancer pain. However, there is currently no sufficient evidence to support the efficacy of a combination of gabapentin and opioids in ameliorating neuropathic cancer pain. Hence, the aim of the present study was to explore the analgesic efficacy of gabapentin combined with opioids in treating neuropathic cancer pain.
\end{abstract}

Methods: PubMed, EMBASE, and Web of Science (Web of Knowledge) were searched for randomized controlled trials and prospective studies via the following keywords: "gabapentin", "opioid”, "cancer", and "neuropathic pain". We used a scale of 0-10 (0 denoting no pain and 10 denoting the worst pain imaginable) to estimate pain intensity and utilized Review Manager 5.3 and Stata12 to analyze data.

Results: Seven studies meeting our criterion were selected from 110 records that were primarily searched. The mean difference of pooled pain intensity and the $95 \%$ confidence interval (CI) was $-1.75(-2.44,-1.07)$ ( $\mathrm{P}$ value $<0.00001$; treatment group versus control group or time to outcome assessment versus baseline). The pain intensity of cancer patients after a combined treatment of gabapentin and opioids was significantly lower than that of patients receiving opioids alone.

Conclusions: Our meta-analysis showed that gabapentin combined with opioids effectively alleviated neuropathic cancer pain compared with that of opioids alone.

Keywords: Cancer pain; neuralgia; gabapentin; analgesics, opioid

Submitted Aug 10, 2020. Accepted for publication Dec 24, 2020.

doi: $10.21037 /$ tcr-20-2692

View this article at: http://dx.doi.org/10.21037/tcr-20-2692

\section{Introduction}

Neuropathic cancer pain is defined as pain resulting from nerve injury or compression by tumor infiltration or anticancer treatment (i.e., chemotherapy, surgery, or radiation therapy), including at least one of the following symptoms or signs referred to the pain area: continuous sensory disturbance (e.g., dysesthesia, hyperesthesia, allodynia, burning pain), or incidental pain (e.g., shooting, lancinating pain) (1-3). Currently, opioid analgesics are regarded as the first-line drugs to alleviate pain intensity. However, opioids are unable to achieve satisfactory efficacies in relieving neuropathic cancer pain due to drug tolerance and adverse effects. Based on the World Health Organization threestep analgesic ladder, anticonvulsant drugs are suggested to be adjuvants to opioids for enhancing analgesic effects and/ 
or reducing the side effects of opioids. Unfortunately, the efficacies of combinations of anticonvulsants and opioids in treating neuropathic cancer pain have not been sufficiently validated.

Gabapentin is an anticonvulsant drug that has been applied in the treatment of painful diabetic neuropathy, postherpetic neuralgia, and other types of neuropathic pain (4-7). At present, the acknowledged mechanism of alleviating neuropathic pain from gabapentin is associated with its functions in binding to $\alpha-2-\delta$ subunits of calcium receptors located in the spinal cord and inhibiting the release of excitatory neurotransmitters (8-11). Meanwhile, the $\alpha-2-\delta$ subunits of voltage-gated $\mathrm{Ca}^{2+}$ channels (VGCCs) play a prominent role in the onset of neuropathic pain (12). Furthermore, by inhibiting $\mathrm{N}$-methyl-D-aspartatereceptor-induced excitotoxicity, gabapentin can also exert partial protective effects against glutamate-induced neuronal injury (13). It has been demonstrated that a combination of gabapentin and opioids significantly inhibits evoked dorsalhorn neuronal responses and relieves pain intensity, while administration of the drug alone lacks such efficacy (14). In terms of drug interactions, some studies have suggested that opioids are able to increase the absorption of gabapentin by reducing intestinal motility, which may partly explain the enhanced analgesic effects of a combination of gabapentin and opioids (15). However, the detailed mechanisms underlying interactions between opioids and gabapentin require further elucidation. Furthermore, the minimal adverse effects of gabapentin have promoted its wide clinical application, which is also an attractive characteristic for its use as an adjunctive analgesic with opioids $(16,17)$.

Some researchers have reported a favorable combination of gabapentin with opioids in terms of relieving neuropathic cancer pain (18). However, other studies have suggested that the efficacy of this combination is not significant (16). Therefore, the current evidence of gabapentin as an adjuvant analgesic to opioids in alleviating neuropathic cancer pain is insufficient. Thus, it is of great importance and necessity to explore the efficacy of gabapentin combined with opioids in treating neuropathic cancer pain. We present the following article in accordance with the PRISMA reporting checklist (available at http://dx.doi.org/10.21037/tcr-20-2692).

\section{Methods}

\section{Search strategy}

PubMed, EMBASE, and Web of Science were searched for applicable studies according to the "PICOS" search principle. The search terms were "gabapentin", "opioid", "cancer", and "neuropathic pain", while "clinical trial" acted as the filter criterion. This literature search was completed in January 2020.

\section{Criteria for screening studies}

\section{Types of studies and interventions}

Randomized clinical trials and prospective studies were included in this meta-analysis, the contents of which were associated with the analgesic evaluation of gabapentin combined with opioids. Case reports, case series, observational studies, meta-analysis, and reviews were excluded. The studies were screened from January 2020 through April 2020.

\section{Types of patients}

The patients included in our present meta-analysis were over 18 years old and were confirmed as cancer patients with neuropathic pain. Patients diagnosed with neuropathic pain had one of the following symptoms or signs: burning pain, shooting (electric-shock-like) pain, or allodynia (19). The medical history, results of examinations and available imaging studies (e.g., computed tomography, magnetic resonance imaging) for each patient were also used to assist investigators to diagnose the type of pain (20). Some of the investigated patients who have previously used other non-opioid analgesic drugs-such as nonsteroidal antiinflammatory drugs (NSAIDs), steroids, antidepressants, anxiolytics, or muscle relaxants-were permitted to be included in the corresponding study if they had a constant therapeutic schedule. All included patients with neuropathic cancer pain took opioids for analgesia by default.

\section{Types of outcomes}

The primary outcome in this meta-analysis was pain intensity evaluated by the Visual Analogue Scale (VAS), Numeric Rating Scale (NRS), or numerical scales designed by the experimental team to assess pain intensity of patients (21). All the different pain intensity was converted to a range of $0-10$, with 0 corresponding to "no pain" and 10 corresponding to "the worst possible pain".

\section{Data extraction}

We first concentrated on data regarding pain intensity at baseline and at the end of each study. The mean pain intensity, standard error, and changes in pain intensity were 
extracted directly or calculated indirectly. Additionally, the doses of gabapentin and opioids, the duration of the study, and the number of participants, as well as the descriptions of the study methods and results, were also recorded. Of note, if a study was categorized as a randomized controlled trial, the number of patients was calculated as the sum of the numbers of patients in the control group and experimental group. If a study was a prospective controlled study, patient number was defined with the minimum value representing the number of patients that completed the study. The quality of each study was accessed by the Newcastle-Ottawa Scale (NOS) (22). The review procedure consisted of two authors independently extracting data and generating dataextraction sheets. Data sheets were then compared and judged by a third review author.

\section{Statistical analysis}

We used Review Manager 5.3 software to pool the mean change of pain intensity, as well as its standard error, across studies. If a study was categorized as a randomized controlled trial, the difference in pain intensity between the control group and experimental group was used to evaluate the analgesic efficacy. If a study was a prospective controlled study, the pain intensity at baseline was compared with that at the end point of the observational window. All data regarding pain intensity was converted into a range from 0 to 10 . Due to the same evaluation criterion, we chose the mean difference mode in Review Manager 5.3 software.

$\mathrm{I}^{2}$ was used to determine the degree of heterogeneity in our meta-analysis. If the heterogeneity was over $50 \%$, the random-effects mode was selected, and the causes for high heterogeneity were explored by subgroup analysis. Otherwise, the fixed-effects mode was chosen. Stata12 software was used to analyze publication bias via a funnel plot, Begg's test, and Egger's test. A P value less than 0.05 was considered statistically significant.

\section{Results}

\section{Literature search and description of studies}

We primarily searched 110 records through PubMed, EMBASE, and Web of Science using keywords described in our Methods. After using EndNote X7 Software to remove duplicates, 29 records were removed, after which 81 records remained. Among the remaining records, 71 records did not meet our criteria after detailed screening. Thus, 10 studies were preliminarily selected for further analysis. However, there were two studies from which we could not extract pain intensity, and one study was excluded as the similar research was updated by the same investigators, the updated study was included in our present meta-analysis. Finally, after excluding these studies, our present meta-analysis contained seven studies with publication dates ranging from 2002 to 2013, including two randomized controlled trials and five prospective controlled studies (Figure 1). The details and quality of each study are shown in Table 1.

\section{Primary outcomes}

After summarizing the mean changes of pain intensity from seven studies, the mean difference and $95 \%$ confidence interval (CI) of neuropathic cancer pain relief were -1.75 $(-2.44,-1.07)$ (Figure 2). The $\mathrm{P}$ value was less than 0.00001 , which indicated that the combination of gabapentin and opioids significantly relieved neuropathic cancer pain compared with that of opioids alone. However, $\mathrm{I}^{2}$ was $99 \%$, showing the high heterogeneity in our present metaanalysis. Thus, subgroup analysis was performed to find the origins of this heterogeneity.

First, we classified the seven included studies based on study types. Two subgroups were established, namely randomized controlled studies and prospective controlled studies, and the $\mathrm{I}^{2}$ values reached $85 \%$ and $98 \%$, respectively. However, the heterogeneity of studies was still at a high level. Different designs and durations in the two randomized controlled studies might be the major influence on heterogeneity. Furthermore, $\mathrm{I}^{2}$ of the prospective controlled studies did not decrease significantly. Thus, we performed a more detailed subgroup analysis based on the number of patients in each study. To some extent, the scale of a study can indirectly reflect its validity and statistical power. The studies containing more than 20 patients at the end of the study observation period were defined as large-scale studies, whereas studies containing less than 20 patients at the end of the study observation period were defined as small-scale studies. The smallscale-study subgroup had much lower heterogeneity $\left(\mathrm{I}^{2}=0 \%\right)$, and the large-scale-study subgroup had higher heterogeneity $\left(\mathrm{I}^{2}=64 \%\right)$ (Figure 3$)$. The heterogeneity of the large-scale-study subgroup was still over $50 \%$, which may have been due to the different designs of studies. However, the decrease in $\mathrm{I}^{2}$ following this subgroup analysis was reduced compared to the $98 \%$ heterogeneity of all prospective controlled studies, indicating that the number 


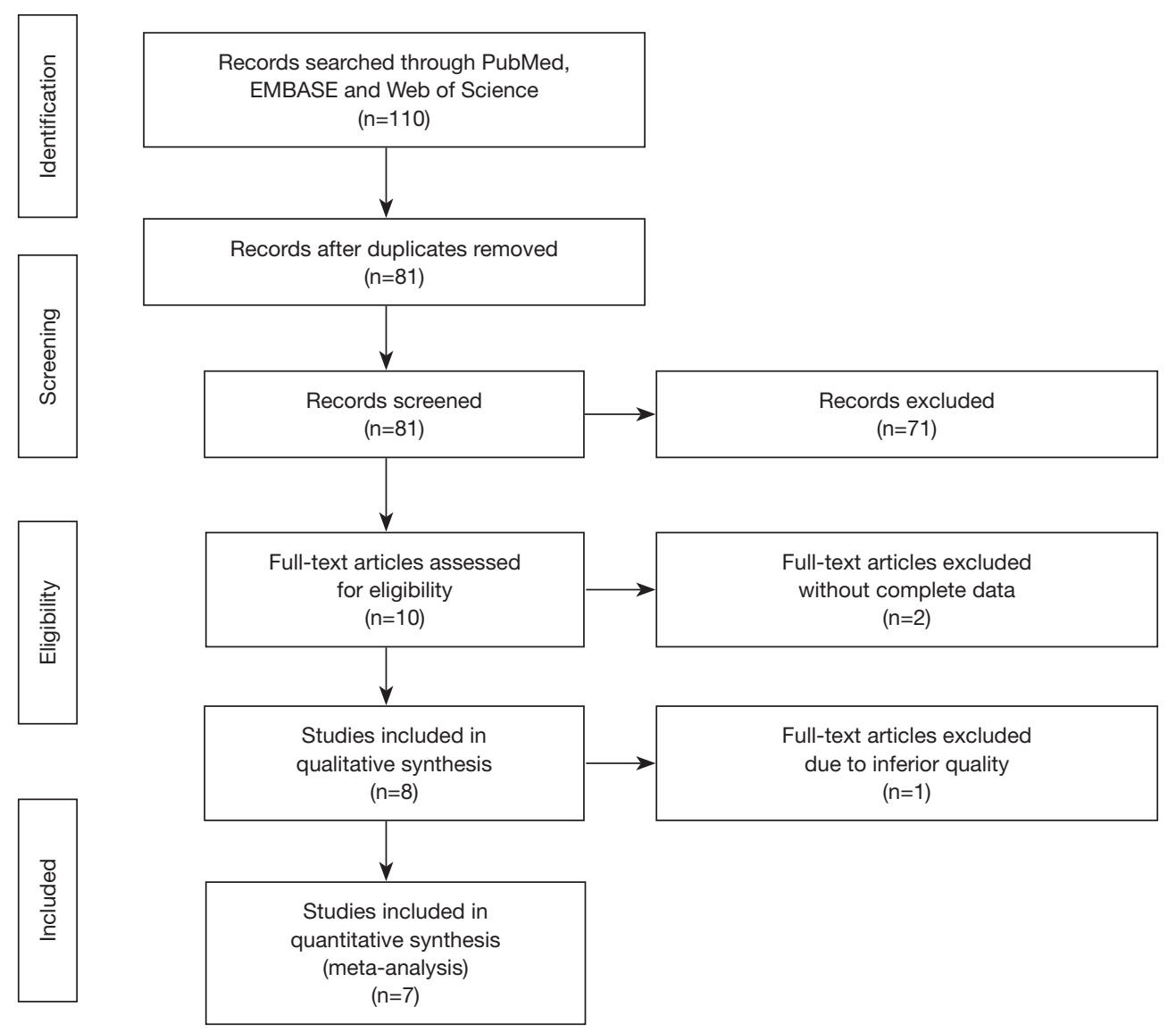

Figure 1 Flow of studies through selection process.

Table 1 Characteristics of included studies

\begin{tabular}{|c|c|c|c|c|c|c|}
\hline First author & Year & Region & Design & Patient number & Gabapentin dose (mg/day) & Quality [0-9] \\
\hline Banerjee (24) & 2013 & India & Open-label parallel group & 40 & $600-1,800$ & 7 \\
\hline Bosnjak (25) & 2002 & Serbia & Open-label single-center group & 15 & $900-1,800$ & 5 \\
\hline Caraceni (26) & 2004 & America & Double-blind parallel group & 89 & $300-1,800$ & 8 \\
\hline Ross (3) & 2005 & United Kingdom & Open-label parallel group & 41 & $400-1,200$ & 6 \\
\hline Takahashi (16) & 2010 & Japan & Open-label single-center group & 20 & $200-2,400$ & 6 \\
\hline
\end{tabular}

of patients was one of the main origins of heterogeneity. There was insufficient data for subgroup analysis based on the types of neuropathic pain induced by anti-cancer treatment or cancer progress, which may be another origin of heterogeneity. Overall, all the $\mathrm{P}$ values were less than 0.05 in the subgroups, suggesting that gabapentin may be an effective adjuvant analgesic to opioids in the treatment of neuropathic cancer pain.

\section{Publication bias}

Stata12 software was used to detect publication bias and 


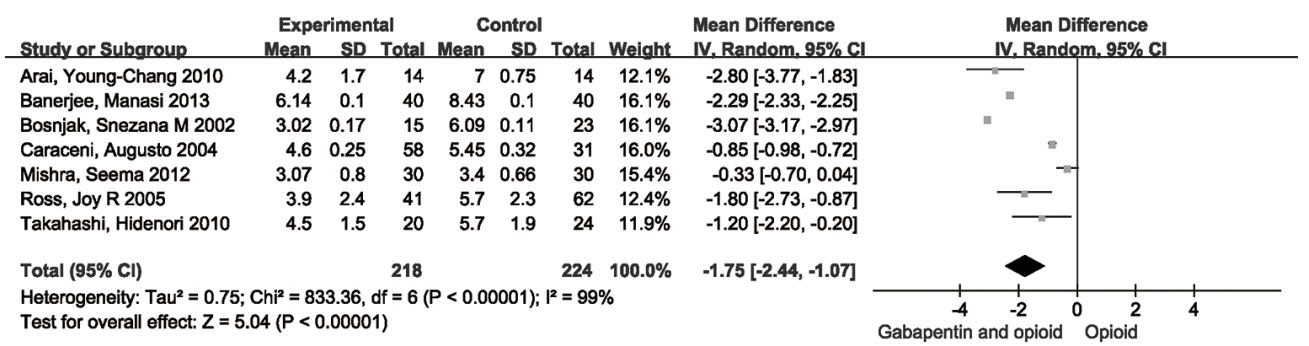

Figure 2 Forest plot of the combination of gabapentin and opioids to alleviate neuropathic cancer pain compared with opioids alone. CI, confidence interval.

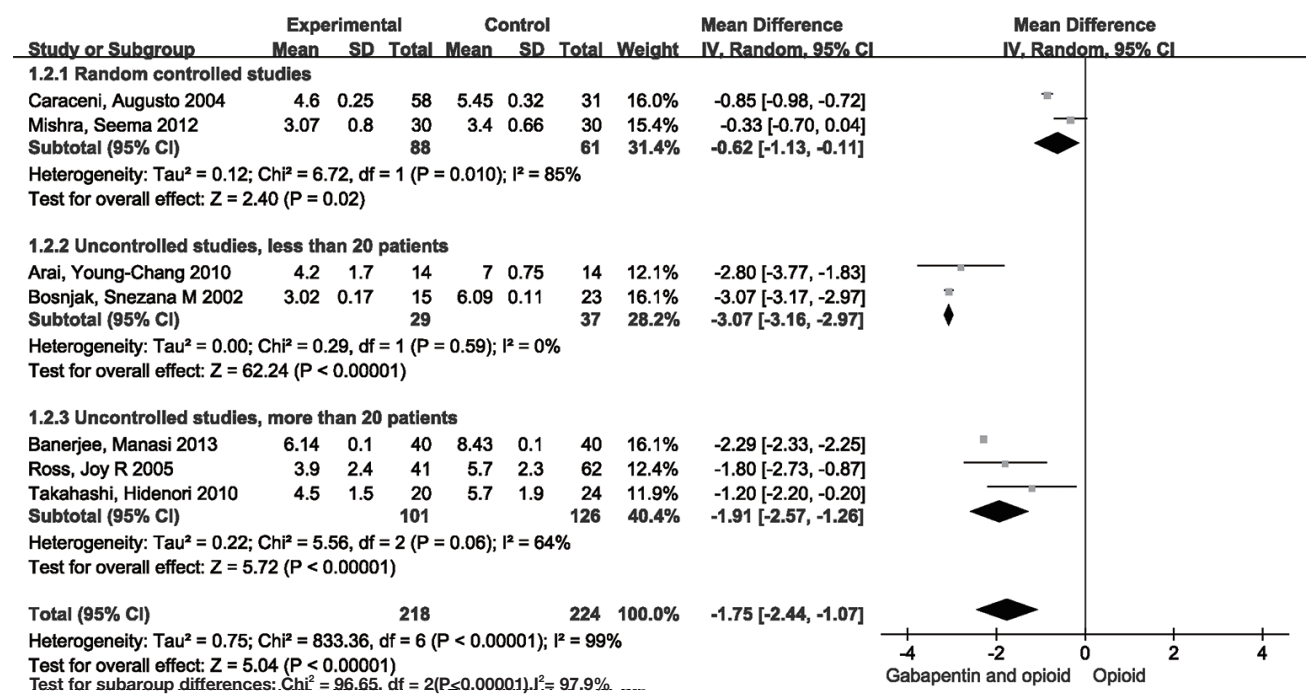

Figure 3 Subgroup analysis based on the number of patients. CI, confidence interval.

heterogeneity via Begg's test and Egger's test. The results of these tests demonstrated that our meta-analysis did not have significant publication bias, such that the $\mathrm{P}$ values of Begg's test and Egger's test (0.55 and 0.56) were each more than 0.05 . For our funnel plot, some points were out of the limits of the $95 \%$ CI, which may have been attributed to high heterogeneity (Figure 4).

\section{Discussion}

Many studies have explored the efficacy of gabapentin combined with opioids in alleviating neuropathic noncancer pain, but evidence to support the efficacy of this combination under cancer conditions has been lacking. Our present meta-analysis analyzed the analgesic efficacy of a combination of gabapentin and opioids for treating neuropathic cancer pain. Our results revealed that this combination significantly alleviated neuropathic cancer pain, such that the mean difference in pain intensity was -1.75 $(-2.44,-1.07)$. Furthermore, subgroup analysis based on the type of study and the number of patients also revealed that a combination of gabapentin and opioids was efficacious in alleviating neuropathic cancer pain. Additionally, our metaanalysis did not possess publication bias, further indicating the validity of our present findings.

However, influences from other non-opioid analgesic drugs-such as NSAIDs, steroids, antidepressants, anxiolytics, or muscle relaxants-may have existed in our present meta-analysis. To the best of our knowledge, there have been few studies that have previously reported definitive interactions of gabapentin with analgesic adjuvants, especially in the context of treating neuropathic cancer pain. First, for NSAIDs, a combination of ibuprofen with gabapentin has been shown to achieve an increased 


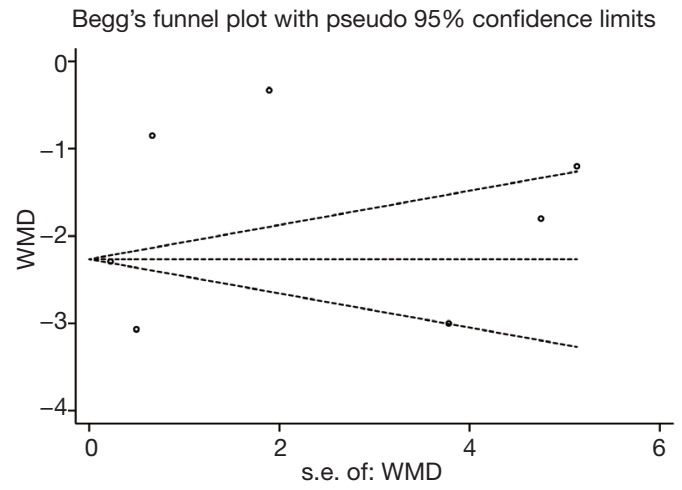

Figure 4 Begg's funnel plot of combination of gabapentin with opioids for neuropathic cancer pain treatment. WMD, weighted mean difference.

efficacy in controlling post-injury pain (28). Moreover, the use of a combination of diclofenac with gabapentin has been shown to be more efficacious and safer than that of monotherapy in treating neuropathic pain in rats (29). Second, for steroids, dexamethasone combined with gabapentin has been shown to relieve pain following tonsil surgery in children (30). In contrast, there has been no report on the efficacy of such a combination in relieving neuropathic cancer pain. However, several studies have suggested favorable effects of pain relief of gabapentin compared with those of steroids, including hydrocortisone and aldosterone (31). In terms of antidepressant drugs, the majority of the research works have focused on comparing gabapentin with other agents in the treatment of pain, pruritus or hot flashes, such as mirtazapine and venlafaxine (32-34). However, few researchers have investigated the interaction of antidepressant drugs or anxiolytics with gabapentin. Taken together, the mechanisms and efficacies of a combination of gabapentin with other non-opioid analgesic drugs require further investigation, especially in the context of treating neuropathic cancer pain.

In our present meta-analysis, neuropathic cancer pain in enrolled patients was not completely controlled with opioids or other analgesic adjuvants. Although some of the selected studies permitted usage of previous medication, the doses remained unchanged throughout the studies. Moreover, based on experimental designs of the included studies, patients in three studies previously used other analgesic adjuvants such as steroids, antidepressants, anxiolytics, or muscle relaxants $(16,23,26)$. Thus, we conducted a two-sample Kolmogorov-Smirnov test based on the mean difference of pain intensity in the above three studies compared with that of the other studies. The $\mathrm{P}$ value of this analysis was 0.89 , indicating a negligible influence of previous medication on pain relief. Despite a potential synergistic effect of gabapentin with other adjuvants, our meta-analysis revealed that gabapentin likely played a dominant role in providing relief from neuropathic cancer pain.

There were also some limitations of our present metaanalysis. The number of collected samples was restricted. Additionally, the heterogeneity was high in this metaanalysis, which may have been caused by differences in experimental designs and the numbers of patients across studies, as indicated by our results of subgroup analysis. We also attempted to perform subgroup analysis based on the types of neuropathic pain induced by anti-cancer treatment or cancer progress, but the data was insufficient due to the small number of studies included in our metaanalysis. Thus, additional high-quality studies on this topic should be gathered to consolidate evidence for a preferable combination of gabapentin with opioids for treating neuropathic cancer pain. Some studies have identified concordance between the clinician diagnosis and pain assessment screening tool outcomes for Neuropathic Symptoms and Signs (LANSS), Douleur Neuropathique en 4 (DN4) or painDETECT (PDQ) in patients with cancer pain (35). Unfortunately, the majority of included studies did not mention the screening tools for the aid of pain diagnosis. Further research is needed to standardize and improve clinical assessments in patients with neuropathic cancer pain (36). Finally, multimodal analgesic efficacy deserves validation and application in the future.

\section{Conclusions}

This meta-analysis supports the enhanced analgesic efficacy of gabapentin combined with opioids, compared with that of opioids alone, in the treatment of neuropathic cancer pain. Hence, our findings may help to further guide clinical treatment of neuropathic cancer pain to improve the quality of life of cancer patients and to inspire researchers to further elucidate the mechanisms underlying the analgesic efficacy of gabapentin combined with opioids.

\section{Acknowledgments}

We thank LetPub (www.letpub.com) for its linguistic assistance during the preparation of this manuscript.

Funding: None. 


\section{Footnote}

Reporting Checklist: The authors have completed the PRISMA reporting checklist. Available at http://dx.doi. org/10.21037/tcr-20-2692

Peer Review File: Available at http://dx.doi.org/10.21037/tcr20-2692

Conflicts of Interest: All authors have completed the ICMJE uniform disclosure form (available at http://dx.doi. org/10.21037/tcr-20-2692). The authors have no conflicts of interest to declare.

Ethical Statement: The authors are accountable for all aspects of the work in ensuring that questions related to the accuracy or integrity of any part of the work are appropriately investigated and resolved.

Open Access Statement: This is an Open Access article distributed in accordance with the Creative Commons Attribution-NonCommercial-NoDerivs 4.0 International License (CC BY-NC-ND 4.0), which permits the noncommercial replication and distribution of the article with the strict proviso that no changes or edits are made and the original work is properly cited (including links to both the formal publication through the relevant DOI and the license). See: https://creativecommons.org/licenses/by-nc-nd/4.0/.

\section{References}

1. Portenoy RK, Lesage P. Management of cancer pain. Lancet 1999;353:1695-700.

2. Cavenagh J, Good P, Ravenscroft P. Neuropathic pain: are we out of the woods yet? Intern Med J 2006;36:251-5.

3. Ross JR, Goller K, Hardy J, et al. Gabapentin is effective in the treatment of cancer-related neuropathic pain: a prospective, open-label study. J Palliat Med 2005;8:1118-26.

4. Backonja M, Beydoun A, Edwards KR, et al. Gabapentin for the symptomatic treatment of painful neuropathy in patients with diabetes mellitus: a randomized controlled trial. JAMA 1998;280:1831-6.

5. Rosenberg JM, Harrell C, Ristic H, et al. The effect of gabapentin on neuropathic pain. Clin J Pain 1997;13:251-5.

6. Rowbotham M, Harden N, Stacey B, et al. Gabapentin for the treatment of postherpetic neuralgia: a randomized controlled trial. JAMA 1998;280:1837-42.
7. Vinik A. CLINICAL REVIEW: Use of antiepileptic drugs in the treatment of chronic painful diabetic neuropathy. J Clin Endocrinol Metab 2005;90:4936-45.

8. Bennett MI, Simpson KH. Gabapentin in the treatment of neuropathic pain. Palliat Med 2004;18:5-11.

9. Field MJ, Oles RJ, Lewis AS, et al. Gabapentin (neurontin) and S-(+)-3-isobutylgaba represent a novel class of selective antihyperalgesic agents. Br J Pharmacol 1997;121:1513-22.

10. Gee NS, Brown JP, Dissanayake VU, et al. The novel anticonvulsant drug, gabapentin (Neurontin), binds to the alpha2delta subunit of a calcium channel. J Biol Chem 1996;271:5768-76.

11. Shimoyama M, Shimoyama N, Hori Y. Gabapentin affects glutamatergic excitatory neurotransmission in the rat dorsal horn. Pain 2000;85:405-14.

12. Alles SRA, Smith PA. Etiology and pharmacology of neuropathic pain. Pharmacol Rev 2018;70:315-47.

13. Kim YS, Chang HK, Lee JW, et al. Protective effect of gabapentin on $\mathrm{N}$-methyl-D-aspartate-induced excitotoxicity in rat hippocampal CA1 neurons. J Pharmacol Sci 2009;109:144-7.

14. Matthews EA, Dickenson AH. A combination of gabapentin and morphine mediates enhanced inhibitory effects on dorsal horn neuronal responses in a rat model of neuropathy. Anesthesiology 2002;96:633-40.

15. Eckhardt K, Ammon S, Hofmann U, et al. Gabapentin enhances the analgesic effect of morphine in healthy volunteers. Anesth Analg 2000;91:185-91.

16. Takahashi H, Shimoyama N. A prospective open-label trial of gabapentin as an adjuvant analgesic with opioids for Japanese patients with neuropathic cancer pain. Int J Clin Oncol 2010;15:46-51.

17. Finnerup NB, Otto M, McQuay HJ, et al. Algorithm for neuropathic pain treatment: an evidence based proposal. Pain 2005;118:289-305.

18. Smith EM, Bakitas MA, Homel P, et al. Preliminary assessment of a neuropathic pain treatment and referral algorithm for patients with cancer. J Pain Symptom Manage 2011;42:822-38.

19. Boureau F, Doubrere JF, Luu M. Study of verbal description in neuropathic pain. Pain 1990;42:145-52.

20. La Cesa S, Tamburin S, Tugnoli V, et al. How to diagnose neuropathic pain? The contribution from clinical examination, pain questionnaires and diagnostic tests. Neurol Sci 2015;36:2169-75.

21. Caraceni A, Cherny N, Fainsinger R, et al. Pain measurement tools and methods in clinical research in 
palliative care: recommendations of an Expert Working Group of the European Association of Palliative Care. J Pain Symptom Manage 2002;23:239-55.

22. Wells GA, Shea B, O'Connell D, et al. The NewcastleOttawa Scale (NOS) for assessing the quality of nonrandomized studies in meta-analysis. Available online: http://www.ohri.ca/programs/clinical_epidemiology/ oxford.htm

23. Arai YC, Matsubara T, Shimo K, et al. Low-dose gabapentin as useful adjuvant to opioids for neuropathic cancer pain when combined with low-dose imipramine. J Anesth 2010;24:407-10.

24. Banerjee M, Pal S, Bhattacharya B, et al. A comparative study of efficacy and safety of gabapentin versus amitriptyline as coanalgesics in patients receiving opioid analgesics for neuropathic pain in malignancy. Indian J Pharmacol 2013;45:334-8.

25. Bosnjak S, Jelic S, Susnjar S, et al. Gabapentin for relief of neuropathic pain related to anticancer treatment: a preliminary study. J Chemother 2002;14:214-9.

26. Caraceni A, Zecca E, Bonezzi C, et al. Gabapentin for neuropathic cancer pain: a randomized controlled trial from the Gabapentin Cancer Pain Study Group. J Clin Oncol 2004;22:2909-17.

27. Mishra S, Bhatnagar S, Goyal GN, et al. A comparative efficacy of amitriptyline, gabapentin, and pregabalin in neuropathic cancer pain: a prospective randomized doubleblind placebo-controlled study. Am J Hosp Palliat Care 2012;29:177-82.

28. Yoon MH, Yaksh TL. Evaluation of interaction between gabapentin and ibuprofen on the formalin test in rats. Anesthesiology 1999;91:1006-13.

29. Ibrahim MA, Abdelzaher WY, Rofaeil RR, et al. Efficacy and safety of combined low doses of either diclofenac or celecoxib with gabapentin versus their single high dose in treatment of neuropathic pain in rats. Biomed Pharmacother 2018;100:267-74.

30. Amin SM, Amr YM. Comparison between preemptive gabapentin and paracetamol for pain control after adenotonsillectomy in children. Anesth Essays Res 2011;5:167-70.

31. Ghadami N, Barzanji A, Nasseri K, et al. Effect of gabapentin in comparison with hydrocortisone on postlaparoscopic cholecystectomy pain control. J Family Med Prim Care 2019;8:652-6.

32. Bordeleau L, Pritchard KI, Loprinzi CL, et al. Multicenter, randomized, cross-over clinical trial of venlafaxine versus gabapentin for the management of hot flashes in breast cancer survivors. J Clin Oncol 2010;28:5147-52.

33. Amr YM, Yousef AA. Evaluation of efficacy of the perioperative administration of Venlafaxine or gabapentin on acute and chronic postmastectomy pain. Clin J Pain 2010;26:381-5.

34. Akhan A, Subasi FD, Bosna G, et al. Comparison of mirtazapine, gabapentin and ondansetron to prevent intrathecal morphine-induced pruritus. North Clin Istanb 2016;3:53-9.

35. Mulvey MR, Boland EG, Bouhassira D, et al. Neuropathic pain in cancer: systematic review, performance of screening tools and analysis of symptom profiles. Br J Anaesth 2017;119:765-74.

36. Park ES, Ahn JM, Jeon SM, et al. Proteomic analysis of the dorsal spinal cord in the mouse model of spared nerve injury-induced neuropathic pain. J Biomed Res 2017;31:494-502.
Cite this article as: Bao H, Wu Z, Wang Q, Wang J, Zhang L, Meng L, Han F. The efficacy of gabapentin combined with opioids for neuropathic cancer pain: a meta-analysis. Transl Cancer Res 2021;10(2):637-644. doi: 10.21037/tcr-20-2692 